\title{
Intrathecal recombinant human 4-sulfatase reduces accumulation of glycosaminoglycans in dura of mucopolysaccharidosis VI cats
}

\author{
Dyane Auclair ${ }^{1}$, John Finnie ${ }^{2}$, Steven U. Walkley ${ }^{3}$, Joleen White ${ }^{4}$, Timothy Nielsen ${ }^{1}$, Maria Fuller ${ }^{1,5}$, Alphonsus Cheng ${ }^{4}$, \\ Charles A. O'Neill ${ }^{4}$ and John J. Hopwood ${ }^{1,5}$
}

INTRODUCTION: Mucopolysaccharidosis VI (MPS-VI) is caused by a deficiency in $\mathrm{N}$-acetylgalactosamine-4-sulfatase activity, resulting in lysosomal accumulation of partially degraded glycosaminoglycans (GAGs). Compressive myelopathy in early-onset MPS-VI patients has been partly attributed to thickening of the dura mater following engorgement with GAG. In this study, we therefore tested whether the dural abnormalities could be prevented in a feline model of the disorder.

RESULTS: All intrathecal injections (IT-INJS) were well tolerated. MPS-VI cats treated with IT-INJ of recombinant human $\mathrm{N}$-acetylgalactosamine-4-sulfatase (rhASB) exhibited reduced vacuolation in the dural fibroblasts, diminished levels of sulfated$\mathrm{N}$-acetylhexosamine $(\mathrm{HNAC}(+\mathrm{S}))$ in the cerebrospinal fluid (CSF) and no hind-limb paresis. Serum anti-rhASB antibodies remained low in MPS-VI cats treated with intravenous enzyme replacement therapy (IV-ERT) and increased slightly in normal cats treated with IT-INJ of rhASB alone. Anti-rhASB antibodies in CSF remained undetectable.

DISCUSSION: These data indicate that repeated IT-INJ of rhASB can safely prevent GAG storage in MPS-VI dura.

METHODS: Cats were assigned to three groups: (i) receiving weekly IV-ERT of rhASB from birth plus six monthly IT-INJS of rhASB from age 2 months; (ii) receiving six monthly IT-INJs of vehicle; or (iii) untreated. Additional normal cats received five fortnightly IT-INJs of rhASB or vehicle alone.

M ucopolysaccharidosis VI (MPS-VI; Maroteaux-Lamy syndrome) is a lysosomal storage disorder caused by a deficiency in $N$-acetylgalactosamine-4-sulfatase (ASB; EC 3.1.6.1) activity. Mutations within the $A S B$ gene produce a loss of functional ASB activity leading to progressive lysosomal accumulation of dermatan and chondroitin sulfates and progressive functional impairment of multiple organs, yielding MPS-VI in humans and mammals, including cats (1). Early-onset MPS-VI patients are usually diagnosed by 2 years of age, and progressive clinical features include major skeletal abnormalities (short stature, dysostosis multiplex), joint stiffness and degenerative joint diseases, cardiomyopathy and valvular dysfunction, upper airway obstruction, and corneal clouding. Death in late childhood to early adulthood often occurs, but patients with lateronset MPS-VI can live into their forties and fifties, usually with progressively worsening symptoms.

Compressive myelopathy leading to leg paresis is common in patients with early-onset MPS-VI consequent to vertebral bone deformities, ligamentum flavum hypertrophy, and/or thickening of the dura mater (2-5). Spinal cord compression in MPS-VI can develop early, and, as with other MPS including MPS-I, MPS-II, and MPS-IV, surgical interventions are often required to alleviate neurological signs (4-7).

Intravenous enzyme replacement therapy (IV-ERT) with recombinant human ASB (rhASB) is now considered a safe therapy for MPS-VI and provides short- and longer-term benefits $(8,9)$. Phase II/III clinical trials showed sustained improvements in walking and climbing endurance in most patients treated with IV-ERT for 97-260 weeks (9). However, these were less pronounced in patients with advanced skeletal disease at the start of the trials (9). Complementary therapeutic approaches require investigation to lessen the risk of developing spinal cord compression and paresis in the most affected MPS-VI patients receiving IV-ERT.

In the MPS-VI cat, IV-ERT from birth effectively improves pathology in various tissues, including heart valves, aorta, bones, liver, and kidney $(10,11)$. Although articular cartilages do not benefit from intravenously administered rhASB due to their avascular nature, they nevertheless show significant improvement when rhASB is injected directly into the joint spaces $(12,13)$. Similarly, neuroparenchyma outside the blood-brain barrier does not benefit from recombinant enzyme administered systemically at a standard dose, with vacuolation evident in dura mater of MPS-VI cats treated weekly with 1 or $2 \mathrm{mg} / \mathrm{kg}$ rhASB from birth to 6 months of age (11). In a pilot study using mature MPS-VI cats tolerant to rhASB, a short course of

\footnotetext{
'Lysosomal Diseases Research Unit, a Research Centre of SA Pathology, North Adelaide, South Australia, Australia; ${ }^{2}$ Veterinary Services Division, SA Pathology, Adelaide, South Australia, Australia; ${ }^{3}$ Sidney Weisner Laboratory of Genetic Neurological Disease, Department of Neuroscience, Rose F. Kennedy Center Albert Einstein College of Medicine, Bronx, New York; ${ }^{4}$ BioMarin Pharmaceutical Inc., Novato, California; ${ }^{5}$ Department of Paediatrics, The University of Adelaide, Adelaide, South Australia, Australia.

Correspondence: John J. Hopwood (john.hopwood@adelaide.edu.au) 
intrathecal therapy was effective at reducing lysosomal storage in dura mater chronically altered by the disease process (14).

In this study, we report the impact of early commencement of intrathecal rhASB therapy in young MPS-VI cats receiving IV-ERT. We also tested whether adverse events develop following repeated intrathecal injections (IT-INJs) of rhASB in immunocompetent normal cats.

\section{RESULTS}

\section{Immune response toward rhASB}

Of eight A/E6 cats, two remained seronegative throughout the study; three had a single low-positive response (dilution factor (DF) <90); three had persistent low anti-rhASB antibody responses (DF ranging between 90 and 810); two N/E5 cats had significantly elevated serum anti-rhASB titers toward the end of therapy (maximum DF: 2430 and 7290); the third N/5 cat remained seronegative; serum anti-rhASB antibody titers in all A/V6, N/V6, and N/E6 cats were below or near the limit of detection of the electrochemiluminescence immunoassay (i.e., DF $\leq 30$ ). All cerebrospinal fluid (CSF) samples exhibited anti-rhASB antibody titers below or near the limit of detection of the electrochemiluminescence immunoassay (i.e., DF $\leq 30$ ).

\section{Safety of IT-INJ}

Repeated IT-INJ and general anesthesia did not affect feline growth (Figure 1). The majority of cats treated with IT-INJ did not show any noteworthy clinical abnormalities during the study: cat N/E5-2 became febrile 1 day after IT-INJ\#3, and cat N/V6-1 had mild neurological deficit after IT-INJ\#5; both animals recovered with supportive treatment and tolerated subsequent IT-INJs without incident. Neither incident was attributed to rhASB administration. Small focal sites of mild Wallerian degeneration, resembling those reported previously (14), were observed in the brainstem and first spinal cord segment near the site of injection across all animal groups receiving IT-INJ of rhASB or vehicle (with or without polysorbate-80). Absence of inflammation in the majority of CSF samples in intrathecally treated cats correlated with the absence of inflammation in the brain, spinal cord, and dura at the end of the study; in a few CSF samples, there were erythrocytes attributed to iatrogenic hemorrhage (red blood count range: $0-282,000 \times 10^{6} / 1$ ). In the CSF sample collected 1 day after IT-INJ\#5 in one normal cat that seroconverted (N/E5-1), there were elevated nucleated cells (including activated macrophages) (nucleated cell count: $\left.280 \times 10^{6} / 1\right)$, reactive pleocytosis, hemosiderophages, and borderline elevation in protein concentration $(0.32 \mathrm{~g} / \mathrm{l})$, which may have been due to slight blood contamination (red blood count: $8,350 \times 10^{6} / 1$ ); however, inflammation was not detected in histological sections of cerebrum, cerebellum, and dura.

\section{Efficacy of IT-INJ}

Measurements of dural thickness showed large intra- and intersample variability. Overall ranges were 92-327, 54-223, and 19-131 $\mu \mathrm{m}$ for the thicker, intermediate, and thinner parts of the sections, respectively. Thicker samples were among the normal cats, and thinner samples were among the A/V6 cats (data not
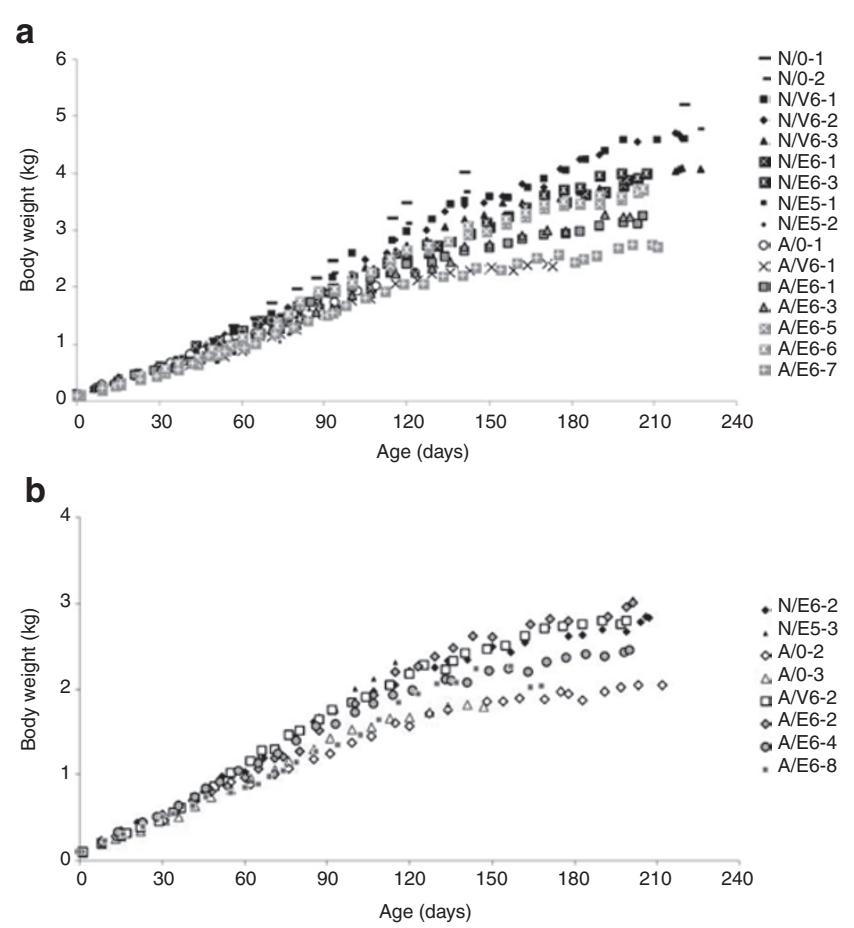

Figure 1. Change in body weight. (a) Males $(-\mathrm{N} / 0-1 ;-\mathrm{N} / 0-2 ;$ - N/V6-1;

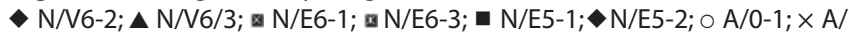

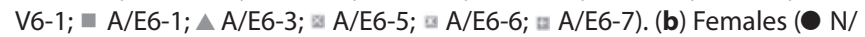
E6-2; $\triangle \mathrm{N} / \mathrm{E} 5-3 ; \diamond \mathrm{A} / 0-2 ; \triangle \mathrm{A} / 0-3 ; \square \mathrm{A} / \mathrm{V6}-2 ; \triangle \mathrm{A} / \mathrm{E6}-2 ;>\mathrm{A} / \mathrm{E} 6-4 ; \square \mathrm{A} / \mathrm{E6}-8)$.

shown). Initial qualitative evaluation (unblended) of the dura confirmed the absence of vacuolation in normals (Figure 2a,b) and indicated a general improvement in tissue appearance in samples obtained from most A/E6 cats compared with A/V6 and $\mathrm{A} / 0$ cats (Figure $2 \mathrm{c}-\mathbf{f}$ ). To confirm these observations, a veterinary histopathologist subsequently examined the dura samples in a blinded manner. A score system rating the extent of vacuolation from 0 to 4 (no to severe vacuolation) showed a marked trend toward less vacuolation in dura samples of A/E6 cats compared with A/V6 or A/0 cats (Table 1 ).

Sulfated- $N$-acetylhexosamine $(\operatorname{HNAc}(+S))$ concentration in CSF indicated a significant group $\times$ time interaction $(P<0.0001)$ for the three groups of cats (i.e., group 1: all normal cats; group 2: A/E6 cats; group 3: (A/V6 + A/0) cats) (Figure 3): the (A/V6 + $\mathrm{A} / 0)$ cats were significantly different $(P<0.0001)$ from the normal cats at all time points; the A/E6 cats were significantly different $(P<0.003)$ from $(\mathrm{A} / \mathrm{V} 6+\mathrm{A} / 0)$ cats at all time points except time 0 ; and A/E6 cats were significantly different $(P<0.05)$ from normal cats at all time points except at 70 days of intrathecal therapy (Figure 2).

A clear reduction in vacuolation was evident in the dura of one MPS-VI cat (A/E6-8) 23 days after IT-INJ\#4 (i.e., up to 107 days of intrathecal therapy when early withdrawal was necessary due to severe anemia/icterus) (Figure $2 \mathrm{~g}$,h) along with low levels of $\mathrm{HNAc}(+\mathrm{S})$ in the final CSF sample (see 107-day time point in Figure 3). Although these observations should be interpreted with caution due to the poor health of the cat, they suggest that substantial re-accumulation of storage did not occur in that period. 

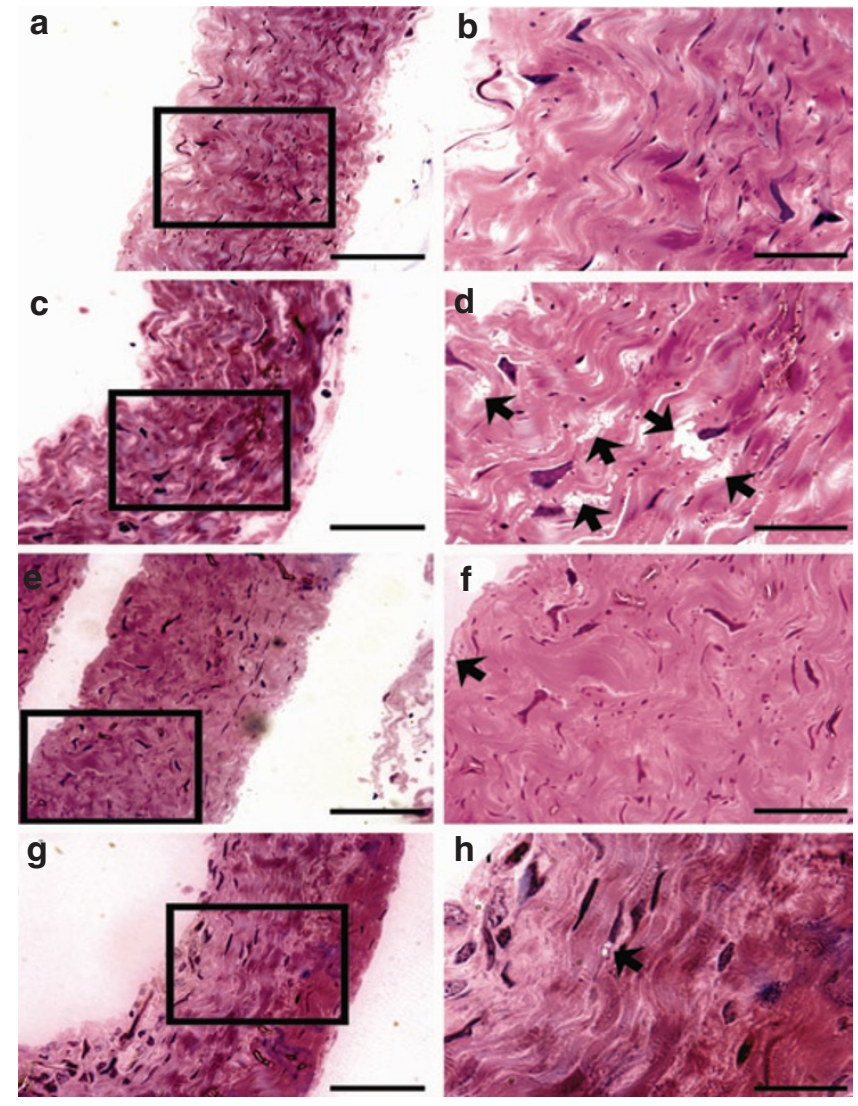

Figure 2. Histological appearance of the dura mater. Dura from normal cat $((\mathbf{a}, \mathbf{b})$ cat N/V6-2) and untreated MPS-VI cat ((c,d) cat A/0-2) resembled that of MPS-VI cats treated with IT-INJ of vehicle (not shown). Treated MPS-VI cat dura 2 days after the final IT-INJ of rhASB $((\mathbf{e}, \mathbf{f})$ cat A/E6-2). Treated MPS-VI cat (sick) dura 23 days after IT-INJ\#4 ((g,h) cat A/E6-8). Black arrowheads show lysosomal vacuoles in dural fibroblasts. Semi-thin $(1 \mu \mathrm{m})$ toluidine blue-stained sections were used. Micrographs $\mathbf{b}, \mathbf{d}, \mathbf{f}$, and $\mathbf{h}$ represent higher magnification (original magnification $\times 1,000$, $\mathrm{bar}=20 \mu \mathrm{m}$ ) of the rectangle area seen on the left micrographs $\mathbf{a}, \mathbf{c}, \mathbf{e}$, and g (original magnification $\times 400$, bar $=50 \mu \mathrm{m}$ ). IT-INJ, intrathecal injection; MPS, mucopolysaccharidosis.

\section{Response to IV-ERT}

No abnormalities were observed during or immediately after each IV-ERT. Cat A/E6-7 was the only male in this group that did not exhibit an overall weight gain (Figure 1a). Body weights of the other four A/E6 males remained lower than normal males from 3.5 months; N/0 males grew slightly better than $\mathrm{N} /$ V6, N/E6, and N/E5 males; A/V6-2 exhibited weight gain similar to normal females (Figure 1b). A/E6-8 grew until 150 days of age, after which signs of lethargy, fever, icterus, and anemia became evident and required early study withdrawal at 167 days of age (i.e., 23 days after IT-INJ\#4); although the Coombs test was positive, hemolytic anemia was excluded at necropsy, and hepatitis and splenitis were recorded. No health problems (unrelated to MPS-VI) or macroscopic pathology was detected in other cats in this study. A/0 and A/V6 developed characteristic features of MPS-VI (dwarfism, coarse facies, joint stiffness, and gait abnormalities). A/0-1 and A/0-2 were euthanized at 137 and 146 days of age, respectively, due to grooming difficulties and paresis. Multiple spinal cord compressions were found in $\mathrm{A} / 0-1$; a single compression site was detected in $\mathrm{A} / 0-2$. Similarly, A/V6-1 was euthanized at 173 days of age (2 days after IT-INJ\#5) due to multiple spinal cord compressions leading to severe paresis and grooming difficulties.

At the study end, no skeletal or joint abnormalities were visible on radiographs of normal cats. The A/0 and A/V6 cats exhibited a moderate degree of generalized epiphyseal dysplasia and osteopenia, with degenerative joint disease resembling the radiographical abnormalities observed in young, untreated MPS-VI cats $(10,11)$. Similarly, the radiographic improvements seen in eight A/E6 cats at the end of the study were comparable to those reported in young IV-ERT MPS-VI cats treated from birth $(10,11)$. Although the dimensions and shape of most long bones and lumbar vertebrae were improved in the A/E6 cats as compared with A/V6 and A/0 cats, no skeletal disease features were completely normalized. Moreover, urinary GAG levels in A/E6 cats were lower than those in A/0 cats and in cat A/V6-1 but did not reach the low levels found in normal cats, confirming previous observations (data not shown) $(10,11)$.

\section{DISCUSSION}

IT-INJ of rhASB was well tolerated with no adverse events related to the enzyme preparation, vehicles or diluent, confirming our previous findings of the safety of IT-INJ in two mature immunotolerized MPS-VI cats (14). Histological examination of dural sections from four different anatomical sites showed an unequivocal tendency toward reduced vacuolation in MPS-VI cats treated with monthly IT-INJ of $0.5 \mathrm{mg} / \mathrm{kg}$ rhASB compared with those treated with IT-INJ with vehicle, untreated MPS-VI cats, and historical MPS-VI cats treated with IV-ERT alone from birth (11). A comparable decrease in dura vacuolation was reported in MPS-I dogs treated at different intervals (i.e., weekly, monthly, or quarterly) with IT-INJ of recombinant human $\alpha-\mathrm{L}$ iduronidase $(15,16)$.

Histological observations of vacuolation matched reductions in CSF HNAc $(+S)$ in MPS-VI cats treated with rhASB IT-INJ. We have reported that $\mathrm{CSF} \operatorname{HNAc}(+\mathrm{S})$ in two MPS-VI cats treated weekly with IT-INJ of rhASB normalized after 1 week of therapy (17).

Although the final observations made in one MPS-VI cat withdrawn from the study 23 days after IT-INJ\#4 need to be interpreted with caution due to its poor health, they suggest that 3 weeks is necessary for substantial re-accumulation of lysosomal storage to occur within the dura. Indeed, reduced vacuolation was observed within this cat's dura, and a low $\mathrm{HNAc}(+\mathrm{S})$ level was measured in the CSF sample collected before necropsy. Thus, the amount of $\mathrm{HNAc}(+\mathrm{S})$ in CSF measures a therapeutic response to this therapy in cats.

In MPS-I dogs treated intrathecally with iduronidase, spinal meningeal GAG storage was reduced by $58-70 \%$ as compared with untreated dogs (15). Three months after the last IT-INJ of iduronidase, no reduction in vacuolation was observed in the neocortical meningeal cells, although a $73 \%$ reduction in GAG in the spinal meninges was still detected $(15,16)$. Although useful for screening and monitoring total urinary GAG, dye 
Table 1. Paresis, spinal compression, and vacuolation in the dura samples collected at necropsy for three groups of cats (normal cats treated with intrathecal injections (IT-INJs) of vehicle or recombinant human N-acetylgalactosamine-4-sulfatase (rhASB), MPS-VI cats treated with IT-INJ of vehicle or left untreated, and MPS-VI cats treated with IT-INJ of rhASB)

\begin{tabular}{|c|c|c|c|c|c|c|c|c|c|c|}
\hline \multirow[b]{2}{*}{ Group } & \multirow[b]{2}{*}{ Treatment $^{\mathrm{a}}$} & \multirow[b]{2}{*}{ Paresis } & \multirow[b]{2}{*}{ Compression } & \multirow[b]{2}{*}{ Site } & \multicolumn{6}{|c|}{ Ratio of dura exhibiting the following vacuolation score ${ }^{b}$} \\
\hline & & & & & 0 & 1 & 2 & 3 & 4 & Uncertain \\
\hline Normal & $\mathrm{N} / \mathrm{V} 6(n=3)$ & No & No & Cerebrum & $9 / 9$ & & & & & \\
\hline \multirow[t]{2}{*}{$(n=9)$} & $\mathrm{N} / \mathrm{E} 6(n=3)$ & & & Thoracic & $9 / 9$ & & & & & \\
\hline & & & & Lumbar & $9 / 9$ & & & & & \\
\hline MPS-VI & $\mathrm{A} / 0(n=1)$ & $(3 / 5)$ & or & Cervical & & & $2 / 3$ & $1 / 3$ & & \\
\hline cats $^{c}$ & & & multiple & Thoracic & & & & $3 / 3$ & & \\
\hline$(n=3)$ & & & $(2 / 3)$ & Lumbar & & & $1 / 3$ & $1 / 3$ & $1 / 3$ & \\
\hline Treated & $\mathrm{A} / \mathrm{E} 6(n=8)$ & No & No & Cerebrum & & $6 / 8$ & $1 / 8$ & $1 / 8$ & & \\
\hline MPS-VI & & & & Cervical & & $8 / 8$ & & & & \\
\hline
\end{tabular}

MPS, mucopolysaccharidosis.

aNN6, normal treated with 6 IT-INJs of vehicle; N/E5, normal treated with 5 IT-INJs of enzyme (rhASB); N/E6, normal treated with 6 IT-INJS of enzyme (rhASB); AN6, MPS-VI treated with 6 IT-INJs of vehicle; A/0, untreated MPS-VI; A/E6, MPS-VI treated with 6 IT-INJs of enzyme (rhASB). 'bScore system ranking the extent of vacuolation: 0 , absent; 1 , mild; 2 , moderate; 3, pronounced; 4, severe; uncertain, unable to score due to poor sample quality. The dura from two untreated MPS-VI cats (A/0-1 and A/0-3) were not scored for vacuolation due to poor fixation. The dura from four normal cats (groups N/0 and N/N5) were not evaluated.

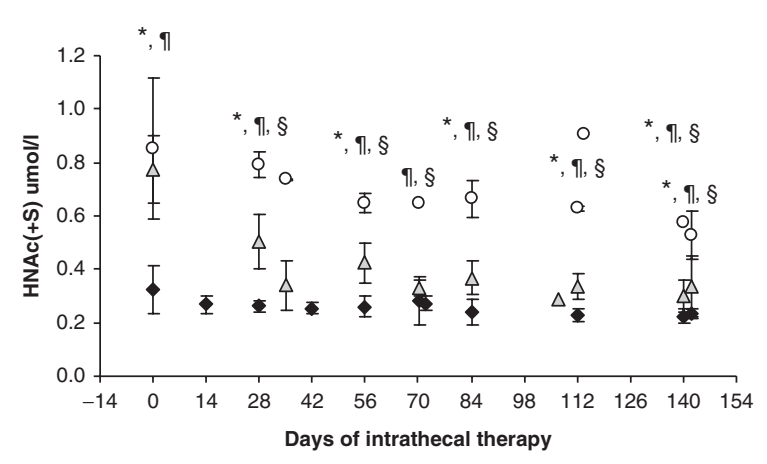

Figure 3. Sulfated monosaccharides in CSF. CSF HNAC(+S) as measured by tandem mass spectrometry. Group differences were tested at $0,28,56,70$, $84,112,140$, and 142 days. Significant difference $(P<0.05)$ between normal cats $(n=1-11)$ and A/E6 cats $(\Delta n=1-8)$ are marked "*". Significant differences $(P<0.05)$ between normal cats and A/V6 and A/0 cats $(0: n=$ $1-3)$ are marked " $₫$ ". Significant differences $(P<0.05)$ between A/E6 cats and $A / V 6$ and $A / 0$ cats are marked " $§$ ". CSF, cerebrospinal fluid; $\mathrm{HNAC}(+S)$, sulfated- $\mathrm{N}$-acetylhexosamine.

methods often lack precision (16) and are impractical for CNS tissue analysis because samples are obtained at necropsy. Measurement of oligosaccharide fragments in CSF by tandem mass spectrometry offers a sensitive and specific approach for monitoring therapeutic efficacy (17-19).

Although lysosomal storage has been reported in a few neurons and glia in the cerebral cortex of MPS-VI cats, CNS impairment is not usually associated with MPS-VI and, accordingly, in this study, the clearance of storage material within neural cells was not examined (20). Consistent with our previous intrathecal therapy study, analysis of dural thickness was inconclusive owing to large intra- and intersample variability, possibly reflecting difficulties in obtaining the correct tissue orientation during embedding and sectioning (14). Nevertheless, the few MPS-VI cats that were not treated intrathecally with rhASB generally had thinner dura than the normal cats, which does not support the hypothesis that dural thickening plays an etiological role in the young feline MPS-VI paresis model. In MPS-VI humans, observations made during diagnostic imaging, surgery, and autopsy have shown that dural thickening at the cervical level can contribute to compressive myelopathy $(2-5)$. Advances in medical imaging techniques have led to major improvements in evaluating dural thickness in humans $(21,22)$.

No spinal cord compression site was seen in any cat treated with IT-INJ and IV-ERT of rhASB, whereas multiple or single spinal cord compression sites were detected in three of five untreated MPS-VI cats. This is consistent with observations that vertebral shapes are improved, and vertebral canal stenosis is prevented, with weekly IV-ERT initiated at an early age. It is possible that the reduction in lysosomal storage within the dura mater leads to improvement in tissue characteristics unrelated to thickness, such as stiffness and elasticity, and that this subsequently prevented the development of compressive force around the spinal cord; however, changes in these parameters remain to be demonstrated.

The low stable level of anti-rhASB antibodies in serum collected at various times before or after systemic and intrathecal administration of rhASB in MPS-VI cats, and the absence of anti-rhASB antibodies in CSF collected before each IT-INJ and at the end of the study in these cats, confirm a state of immune tolerance toward rhASB similar to that observed in other MPS-VI cats treated with IV-ERT alone from birth 
$(10,11)$. Hypersensitivity reactions at the time of IV-ERT and/or at monthly IT-INJ of rhASB were not observed in the MPS-VI cats, indicating that, together with antibody data, the combined IV-ERT and IT-INJ therapy is safe. The capacity to quickly mount an immune response toward intrathecally administered rhASB was seen in two/three normal cats, supporting our previous observation that some immunocompetent normal cats do not develop anti-rhASB antibodies following exposure to rhASB (14). In two normal cats that seroconverted, the humoral immune response mounted within 2 months was mild, indicated by a relatively low elevation in serum anti-rhASB antibodies, the absence of CSF anti-rhASB antibodies, the absence of inflammation in the CNS tissues, and the few inflammatory cells detected in only one CSF sample obtained after IT-INJ\#5. It is possible that the immune response might have intensified over time and that adverse events (such as meningitis) might have developed if fortnightly IT-INJs of rhASB had been continued over the following months in these normal cats. MPS-I dogs treated intrathecally with recombinant human iduronidase developed serum and CSF antibodies against the enzyme, as well as meningitis $(15,16)$. Similarly, intrathecally treated MPS-IIIA dogs developed antibodies against recombinant human sulfamidase, as well as meningitis (23), whereas MPS-IIIA mice treated with repeated IT-INJs of the same enzyme developed circulating antibodies without meningitis or adverse reactions (24-26).

The small, focal, Wallerian-type degeneration observed near the site of IT-INJ in most intrathecally treated cats, irrespective of presence or absence of polysorbate- 80 , was attributed to mild iatrogenic trauma produced during IT-INJ, as previously reported (14), again highlighting the importance of a good injection technique (14). The lumbar region (L4-L5 space) has been used for IT-INJ of recombinant enzyme in MPS-I and MPS-VI patients $(27,28)$; ultrasound guidance will likely be used to localize the subarachnoid space for precise delivery of enzyme into the CSF. The phenotypic improvements observed in the rhASB-treated MPS-VI cats in this study, such as increased bone length, reduced kyphosis, and improved facial appearance were attributed to the early initiation of weekly IV-ERT, again confirming its efficacy $(10,11)$. Even when weekly IV-ERT of $1 \mathrm{mg} / \mathrm{kg}$ rhASB is commenced at birth, some features of MPS-VI remained detectable, e.g., shorter, stiff neck and mild facial abnormalities, and some variations in response are observed due to phenotypic variation. As expected, corneal clouding and degenerative joint diseases continued to develop in the IV-ERT MPS-VI cats due to poor vascularization of these sites. This study has demonstrated that local injections of rhASB benefit tissues not normally accessed by systemically administered rhASB. Indeed, direct injection of rhASB into the CSF bypasses the blood-brain barrier and improves the appearance of the dura mater. Similarly, direct injection of rhASB into joint spaces circumvents the lack of vascularization and improves the appearance of the articular cartilage $(12,13)$.

In summary, monthly IT-INJ of rhASB was well tolerated in MPS-VI cats receiving weekly IV-ERT from an early age and led to substantial reductions in lysosomal storage within the dura mater and $\mathrm{HNAc}(+\mathrm{S})$ concentration in serial CSF samples. These results were similar to those found in mature immunotolerized MPS-VI cats (14) and support the contention that repeated IT-INJ is a safe and efficacious means of regularly administering enzyme into the CNS in MPS-VI and other lysosomal storage disorders for which development of paresis needs to be prevented $(6,7)$ or in which brain function is impaired $(16,23-25,28,29)$.

\section{METHODS}

\section{Enzyme Preparation}

BioMarin Pharmaceutical (Novato, CA) provided rhASB (14). The vehicle solution used with rhASB for monthly IT-INJ contained $10 \mathrm{mM}$ sodium phosphate and $150 \mathrm{mM}$ sodium chloride with $0.025 \%(\mathrm{vol} / \mathrm{vol})$ polysorbate- 80 . A second vehicle solution without polysorbate- 80 was used for fortnightly IT-INJs. The $\mathrm{pH}$ of rhASB and vehicle preparations was 5.8. The concentration of rhASB was $1 \mathrm{mg} / \mathrm{ml}$ and $5 \mathrm{mg} / \mathrm{ml}$ for IV-ERT and IT-INJ, respectively. Before each IT-INJ, one volume of rhASB (or equivalent volume of vehicle) was diluted with two volumes of Elliott's B solution (QOL Medical, Seattle, WA).

\section{Test Animals}

Cats were bred and maintained at the Institute of Medical and Veterinary Science, Adelaide, South Australia, as previously described $(10,11)$. All studies were reviewed and approved by the Women's and Children's Hospital and Institute of Medical and Veterinary Science animal ethics committees.

\section{MPS-VI Diagnosis}

Histological assessment of blood smears at birth and genotyping were performed as described $(11,14,18)$. The severe clinical MPS-VI phenotype (L476P homozygous) used in this study is associated with a low level of feline ASB protein (18).

\section{IV-ERT, IT-INJ, and Sampling}

Eight MPS-VI (A/E6) and three normal (N/E6) cats received weekly IV-ERT of $1 \mathrm{mg} / \mathrm{kg}$ rhASB usually starting within the first 24 hours after birth, plus a total of six IT-INJs of $0.5 \mathrm{mg} / \mathrm{kg}$ rhASB administered once monthly from 2 months of age. IT-INJs were given via the cisterna cerebellomedullaris (cisterna magna). No other cat received IV-ERT. Three normal cats (N/E5) received a total of five IT-INJs of rhASB at 2-week intervals from 2 months of age. Three normal (N/V6) and two MPS-VI cats (A/V6) received a total of six IT-INJs of vehicle (containing polysorbate-80), once per month from 2 months of age. Two additional normal cats received a total of five IT-INJs of vehicle (without polysorbate-80) (N/V5) at 2-week intervals from 7 months of age. Three untreated MPS-VI (A/0) and two normal kittens (N/0) were controls. The IT-INJ technique used was similar to that described with all IT-INJs administered under general anesthesia (14). Each IT-INJ was administered at $117-285 \mu \mathrm{l} / \mathrm{min}$ over a $3-5$ min period. A treatment summary is presented in Table 2. CSF samples were collected before each IT-INJ and before euthanasia (i.e., 1-3 days after the last IT-INJ). Blood was collected before IV-ERT and IT-INJ, and occasionally minutes after the IT-INJ. From 6 weeks of age onward, antihistamines were administered at least $30 \mathrm{~min}$ prior to each IV-ERT (13).

Health assessments included regular monitoring for hypersensitivity reactions, weight gain, gait abnormalities, blood and urine analysis, and final standardized radiographs (14).

\section{Analysis of CSF Samples and Cytology}

CSF was collected and processed for immediate cytospin, cell count, smear evaluation, and measurement of protein concentration, antibody titers, and oligosaccharide fragments (14). Anti-rhASB antibodies in serum and CSF were detected using a bridged electrochemiluminescence 
Table 2 Summary of treatment

\begin{tabular}{|c|c|c|c|c|c|}
\hline Group & $n$ & Phenotype & $\begin{array}{l}\text { IV-ERT' } \\
\text { (dose of } \\
\text { rhASB) }\end{array}$ & $\begin{array}{l}\text { Type and number } \\
\text { of IT-INJs (dose } \\
\text { of rhASB, if } \\
\text { applicable) }\end{array}$ & $\begin{array}{l}\text { Frequency } \\
\text { of IT-INJs }\end{array}$ \\
\hline $\mathrm{N} / 0$ & 2 & Normal & None & None & NA \\
\hline N/V6 & 3 & Normal & None & 6 IT-INJs of vehicle & Monthly \\
\hline N/V5 & 2 & Normal & None & 5 IT-INJs of vehicle & Fortnightly \\
\hline N/E5 & 3 & Normal & None & $\begin{array}{l}5 \text { IT-INJs of rhASB } \\
(0.5 \mathrm{mg} / \mathrm{kg})\end{array}$ & Fortnightly \\
\hline N/E6 & 3 & Normal & $\begin{array}{l}\text { IV-ERT } \\
(1 \mathrm{mg} / \mathrm{kg})\end{array}$ & $\begin{array}{l}6 \text { IT-INJs of rhASB } \\
(0.5 \mathrm{mg} / \mathrm{kg})\end{array}$ & Monthly \\
\hline A/E6 & 8 & MPS-VI & $\begin{array}{l}\text { IV-ERT } \\
(1 \mathrm{mg} / \mathrm{kg})\end{array}$ & $\begin{array}{l}6 \text { IT-INJs of rhASB } \\
(0.5 \mathrm{mg} / \mathrm{kg})\end{array}$ & Monthly \\
\hline$A / V 6$ & 2 & MPS-VI & None & 6 IT-INJs of vehicle & Monthly \\
\hline $\mathrm{A} / 0$ & 3 & MPS-VI & None & None & NA \\
\hline
\end{tabular}

IT-INJ, intrathecal injection; IV-ERT, intravenous enzyme replacement therapy; MPS, mucopolysaccharidosis; NA, not applicable.

a|V-ERT was given over a short period (2-10 min) using an undiluted preparation

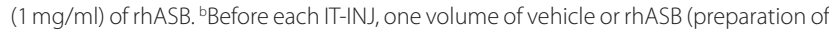
$5 \mathrm{mg} / \mathrm{ml}$ ) was added to two volumes of Elliott's B solution (QOL Medical, Seattle, WA).

immunoassay as described previously $(14,30)$. Titers were reported as the maximum sample DF that yields a positive result. Samples that were not confirmed positive at the minimum DF of 30 were indicated as being below the limit of detection. Differences in titers within 10-fold were not considered significant.

\section{$\mathrm{HNAC}(+\mathrm{S})$ in CSF and Statistical Analysis}

$\mathrm{HNAc}(+\mathrm{S})$ was determined in CSF as described (17). A linear mixed model was run on the CSF levels over time, with group, time, and the interaction as predictors. A compound symmetric covariance structure was used. Before running the full model, a test of the equality of two groups of normal cats (group (N/V6 + N/V5 + N/0) and group (N/E5 + N/E6)) was run. Once equality was established, the mixed model was run with the two normal groups collapsing into one single group: group 1. The A/E6 cats formed group 2; the A/0 and A/V6 cats formed group 3. Group differences were tested at 0 , $28,56,70,84,112,140$, and 142 days, and the $P$ values for these comparisons were adjusted for multiple comparisons using Holm's stepdown procedure (31).

\section{Urinary Glycosaminoglycan (GAG)}

Urine was collected at least monthly, and total GAG was analyzed (13).

\section{Euthanasia, Tissue Collection, and Pathological Evaluation}

Most IT-INJ-treated cats ( 14 of 21) were euthanized at 7 months of age (1-3 days after their last IT-INJ) using a barbiturate overdose. However, cat A/E6-8 was euthanized at 5 months of age due to severe anemia/ icterus; cat A/V6-1 was euthanized at 6 months of age due to severe paresis; three N/E5 cats were euthanized at 4 months of age; and two $\mathrm{N} / \mathrm{V} 5$ cats were euthanized at 9 months of age. Cats A/0-1, A/0-2, and $\mathrm{A} / 0-3$ were euthanized at $4.5,7$, and 5 months of age, respectively. A wide range of tissues was collected, including brain, spinal cord, and dura mater from four levels (cerebrum, cervical, thoracic, and lumbar). Tissues were fixed in $10 \%$ buffered formalin and paraffin embedded, and $6-\mu \mathrm{m}$ sections were cut and stained with hematoxylin and eosin. Two veterinary pathologists independently examined the sections. Dura samples were processed and embedded in Epon-Araldite, and semi-thin $(1 \mu \mathrm{m})$ toluidine blue-stained sections were evaluated for clearance of lysosomal storage $(14,18)$.

\section{ACKNOWLEDGMENTS}

We thank Mark Haskins, University of Pennsylvania, for heterozygous MPS$\mathrm{VI}$ cats and the animal care staff at the IMVS for the care of the colony. We are grateful for regular advice from Allison Crawley (LDRU, SA Pathology) regarding the cat model. We thank Lyn Waterhouse and Ruth Williams (Adelaide Microscopy, University of Adelaide) and the Histopathology Department at SA Pathology for tissue preparation and Nancy Briggs, Public Health Research Unit (Child, Youth and Women's Health Service), for statistical analysis.

\section{STATEMENT OF FINANCIAL SUPPORT}

The work described in this article was supported in part by funding from BioMarin Pharmaceutical, Inc.

Disclosure: J.W., A.C., and C.A.O'N. are employees of BioMarin Pharmaceutical, Inc. BioMarin provided speaker's honoraria and travel support to Dyane Auclair, John Hopwood, and Steven Walkley. BioMarin supplied the recombinant enzyme and was not involved in the study design or interpretation of data.

\section{REFERENCES}

1. Neufeld EF, Muenzer J. The mucopolysaccharidoses. In: Scriver C, Beaudet AL, Sly WS, Valle D, eds. The Metabolic Basis of Inherited Disease. New York: McGraw-Hill, 1989:1565-1587.

2. Sostrin RD, Hasso AN, Peterson DI, Thompson JR. Myelographic features of mucopolysaccharidoses: a new sign. Radiology 1977;125:421-4.

3. Wald SL, Schmidek HH. Compressive myelopathy associated with type VI mucopolysaccharidosis (Maroteaux-Lamy syndrome). Neurosurgery 1984;14:83-8.

4. Vougioukas VI, Berlis A, Kopp MV, Korinthenberg R, Spreer J, van Velthoven V. Neurosurgical interventions in children with MaroteauxLamy syndrome. Case report and review of the literature. Pediatr Neurosurg 2001;35:35-8.

5. Mut M, Cila A, Varli K, Akalan N. Multilevel myelopathy in MaroteauxLamy syndrome and review of the literature. Clin Neurol Neurosurg 2005; 107:230-5.

6. Kulkarni MV, Williams JC, Yeakley JW, et al. Magnetic resonance imaging in the diagnosis of the cranio-cervical manifestations of the mucopolysaccharidoses. Magn Reson Imaging 1987;5:317-23.

7. Kachur E, Del Maestro R. Mucopolysaccharidoses and spinal cord compression: case report and review of the literature with implications of bone marrow transplantation. Neurosurgery 2000;47:223-8; discussion 228-9.

8. Harmatz P, Giugliani R, Schwartz I, et al.; MPS VI Phase 3 Study Group. Enzyme replacement therapy for mucopolysaccharidosis VI: a phase 3, randomized, double-blind, placebo-controlled, multinational study of recombinant human $\mathrm{N}$-acetylgalactosamine 4-sulfatase (recombinant human arylsulfatase B or rhASB) and follow-on, open-label extension study. J Pediatr 2006;148:533-9.

9. Harmatz P, Giugliani R, Schwartz IV, et al.; MPS VI Study Group. Longterm follow-up of endurance and safety outcomes during enzyme replacement therapy for mucopolysaccharidosis VI: final results of three clinical studies of recombinant human $\mathrm{N}$-acetylgalactosamine 4-sulfatase. Mol Genet Metab 2008;94:469-75.

10. Crawley AC, Niedzielski KH, Isaac EL, Davey RC, Byers S, Hopwood JJ. Enzyme replacement therapy from birth in a feline model of mucopolysaccharidosis type VI. J Clin Invest 1997;99:651-62.

11. Auclair D, Hopwood JJ, Brooks DA, Lemontt JF, Crawley AC. Replacement therapy in mucopolysaccharidosis type VI: advantages of early onset of therapy. Mol Genet Metab 2003;78:163-74.

12. Auclair D, Hopwood JJ, Lemontt JF, Chen L, Byers S. Long-term intraarticular administration of recombinant human $\mathrm{N}$-acetylgalactosamine4-sulfatase in feline mucopolysaccharidosis VI. Mol Genet Metab 2007;91:352-61.

13. Auclair D, Hein LK, Hopwood JJ, Byers S. Intra-articular enzyme administration for joint disease in feline mucopolysaccharidosis VI: enzyme dose and interval. Pediatr Res 2006;59(4 Pt 1):538-43.

14. Auclair D, Finnie J, White J, et al. Repeated intrathecal injections of recombinant human 4-sulphatase remove dural storage in mature 
mucopolysaccharidosis VI cats primed with a short-course tolerisation regimen. Mol Genet Metab 2010;99:132-41.

15. Kakkis E, McEntee M, Vogler C, et al. Intrathecal enzyme replacement therapy reduces lysosomal storage in the brain and meninges of the canine model of MPS I. Mol Genet Metab 2004;83:163-74.

16. Dickson P, McEntee M, Vogler C, et al. Intrathecal enzyme replacement therapy: successful treatment of brain disease via the cerebrospinal fluid. Mol Genet Metab 2007;91:61-8.

17. Crawley A, Ramsay SL, Byers S, Hopwood J, Meikle PJ. Monitoring dose response of enzyme replacement therapy in feline mucopolysaccharidosis type VI by tandem mass spectrometry. Pediatr Res 2004;55:585-91.

18. Crawley AC, Yogalingam G, Muller VJ, Hopwood JJ. Two mutations within a feline mucopolysaccharidosis type VI colony cause three different clinical phenotypes. J Clin Invest 1998;101:109-19.

19. Meikle PJ, Fuller M, Hopwood JJ. Mass spectrometry in the study of lysosomal storage disorders. Cell Mol Biol (Noisy-le-grand) 2003;49:769-77.

20. Kuchiwaki H, Inao S, Ishii N, Ogura Y, Gu SP. Human dural thickness measured by ultrasonographic method: reflection of intracranial pressure. J Ultrasound Med 1997;16:725-30.

21. Grassi R, Cavaliere C, Cozzolino S, et al. Small animal imaging facility: new perspectives for the radiologist. Radiol Med 2009;114:152-67.

22. Maikos JT, Elias RA, Shreiber DI. Mechanical properties of dura mater from the rat brain and spinal cord. J Neurotrauma 2008;25:38-51.

23. Hemsley KM, Norman EJ, Crawley AC, et al. Effect of cisternal sulfamidase delivery in MPS IIIA Huntaway dogs. Mol Gen Met 2009;98:383-92.
24. Hemsley KM, Luck AJ, Crawley AC, et al. Examination of intravenous and intra-CSF protein delivery for treatment of neurological disease. Eur J Neurosci 2009;29:1197-214.

25. Hemsley KM, Beard H, King BM, Hopwood JJ. Effect of high dose, repeated intra-cerebrospinal fluid injection of sulphamidase on neuropathology in MPS IIIA mice. Genes Brain Behav 2008;7:740-53.

26. Hemsley KM, King B, Hopwood JJ. Injection of recombinant human sulfamidase into the CSF via the cerebellomedullary cistern in MPS IIIA mice. Mol Genet Metab 2007;90:313-28.

27. Munoz-Rojas MV, Vieira T, Costa R, et al. Intrathecal enzyme replacement therapy in a patient with mucopolysaccharidosis type I and symptomatic spinal cord compression. Am J Med Genet A 2008;146A: 2538-44.

28. Muñoz-Rojas MV, Horovitz DD, Jardim LB, et al. Intrathecal administration of recombinant human $\mathrm{N}$-acetylgalactosamine 4-sulfatase to a MPS VI patient with pachymeningitis cervicalis. Mol Genet Metab 2010;99:346-50.

29. Walkley SU, Thrall MA, Haskins ME, et al. Abnormal neuronal metabolism and storage in mucopolysaccharidosis type VI (Maroteaux-Lamy) disease. Neuropathol Appl Neurobiol 2005;31:536-44.

30. White JT, Martell LA, Van Tuyl A, et al. Development, validation, and clinical implementation of an assay to measure total antibody response to naglazyme (galsulfase). AAPS J 2008;10:363-72.

31. Holm S. A simple sequentially rejective multiple test procedure. Scand J Stat 1979;6:65-70 